\title{
Los docentes como investigadores de su práctica
}

\author{
Yazmín Alejandra Lara Gutiérrez, \\ Adriana Olvera López y \\ Maura Pompa Mansilla
}

\begin{abstract}
Resumen
El presente artículo expresa la experiencia de los participantes en el diplomado "Investigar en educación desde la práctica docente", desarrollado por la Subdirección de Investigación en Educación, perteneciente a la Coordinación de Desarrollo Educativo e Innovación Curricular (CODEIC) de la Universidad Nacional Autónoma de México (UNAM). Dicho diplomado se realizó a lo largo del 2017: su diseño durante el primer semestre, y su impartición y evaluación a lo largo de la segunda mitad de ese año. En el texto se pueden encontrar las voces tanto de los docentes que cursaron el programa como de quienes estuvieron a cargo del proceso formativo, desde el diseño de la propuesta e impartición, hasta su evaluación. Los testimonios coinciden en que fue una experiencia de formación significativa y útil para el desarrollo profesional y personal de los involucrados.
\end{abstract}

Palabras clave: formación en investigación, docentes investigadores, investigación en educación, investigación desde la práctica.

\section{TEACHERS AS RESEARCHERS OF THEIR PRACTICE}

\begin{abstract}
This article sets forth the experience of the participants in the specialization course "Research in Education from the Teaching Practice", developed by the Subdirectorate of Research in Education, from the Coordination of Educational Development and Curricular Innovation (CODEIC) of the National Autonomous University of Mexico (UNAM). This course was held throughout 2017, designed during the first semester of that year, and carried out and evaluated throughout the second half of that year. In this document, the voices of both the teachers who took the course and those who were in charge of the training process can be found, parting from the design of the proposal and its implementation, to its evaluation. The testimonies coincide in establishing that it was a significant and useful training experience for their professional and personal development.
\end{abstract}

Key words: research training, teacher researchers, research in education, research from practice.

DOI: http://doi.org/10.22201/codeic.16076079e.2018.v19n4.a5 


\section{Yazmín Alejandra Lara Gutiérrez}

yazminlarag@unam.mx

Universidad Nacional Autónoma de México. Jefa del Departamento de Investigación en Educación, Coordinación de Desarrollo Educativo e Innovación Curricular. Licenciada y doctorante en Psicología. Investigadora en temas relacionados con la inclusión de tecnologías para el aprendizaje y el conocimiento. Docente de educación media superior y superior en sistemas presenciales y a distancia. ORCID iD: http://orcid.org/0000-0002-8915-4748.

\section{Adriana Olvera López}

adriana.olvera@unam.mx

Universidad Nacional Autónoma de México. Coordinadora de Investigación en Educación, Coordinación de Desarrollo Educativo e Innovación Curricular. Licenciada en Economía y maestra en Estudios Latinoamericanos. Ha trabajado en el sector educativo desde hace 17 años como investigadora y docente en licenciatura y posgrado. Se desempeña en temas de formación y evaluación docente y en la educación de jóvenes en el nivel medio superior. ORCID iD: http://orcid. org/0000-0002-7790-3801.

\section{Maura Pompa Mansilla}

maurapompa@unam.mx

Universidad Nacional Autónoma de México. Jefa del departamento de Investigación Traslacional en Educación, Coordinación de Desarrollo Educativo e Innovación Curricular. Licenciada en Pedagogía (Facultad de Filosofía y Letras, UNAM). Ha trabajado desde hace 14 años en instituciones del sector educativo. Se ha desempeñado en el área de evaluación y primordialmente como investigadora en temas relacionados con educación indígena, evaluación y desempeño docente, inter y multiculturalidad, e inclusión de TIC en el aula, entre otros. ORCID iD: http://orcid.org/0000-00027188-1090.

\section{Introducción}

A través de este artículo se da a conocer la experiencia de los participantes en el diplomado "Investigar en educación desde la práctica docente", desarrollado por la Subdirección de Investigación en Educación, perteneciente a la Coordinación de Desarrollo Educativo e Innovación Curricular (CODEIC) de la Universidad Nacional Autónoma de México (UNAM). Es un proceso formativo que está a favor de la construcción social de aprendizajes y del intercambio de experiencias docentes; en él los profesores desarrollan un protocolo de investigación que tiene incidencia en sus prácticas educativas. El artículo versa sobre el surgimiento del diplomado, su fundamentación teórica, la estructura y la experiencia de haberlo cursado, en voz de los docentes que lo cursaron y de las asesoras que diseñaron el diplomado y coordinaron las sesiones. 


\section{El surgimiento del diplomado}

Actualmente los profesores de los niveles de educación media superior y superior enfrentan diversos retos en su quehacer cotidiano, donde, si bien es cierto que en la mayoría de las ocasiones apelan a su formación y experiencia profesional para ofrecer soluciones, suelen también enfrentar dificultades que los rebasan. Lo anterior hace frecuente que busquen alternativas y herramientas para solucionar dichos retos, que pocas veces se resuelven sólo con su experiencia en el ejercicio de la docencia, y que, en lo ideal, deberían implicar a la investigación científica, misma que les puede ofrecer elementos confiables y orientarlos en la toma de decisiones encaminadas a la mejora de su práctica educativa, y la de otros.

Consiente de dichos desafíos, la Subdirección de Investigación en Educación de la CODEIC generó la propuesta del diplomado "Investigar en educación desde la práctica docente", misma que, más que pretender la formación de especialistas en educación, aspiró a propiciar la reflexión crítica de la investigación en educación como una oportunidad para incidir en la práctica docente cotidiana, y en la promoción de la participación colectiva de los profesores en la generación de conocimiento científico.

El propósito fue recoger su experiencia y opinión del diplomado como proceso de formación, en cuanto a diversos ejes...
El diplomado promovió, entonces, que los profesores fueran capaces de ubicar, consultar y poner en funcionamiento investigación útil para su práctica, para afrontar los retos y situaciones cotidianas. Al mismo tiempo, se fomentó que los profesores consideraran aportaciones de la investigación para su práctica reflexiva y crítica; y sobre todo, posibilitó la reducción de la brecha existente entre investigación y docencia, dado que los docentes lograron despertar su interés por convertirse en generadores de investigación educativa, y no sólo quedarse en el papel de receptores y consumidores del conocimiento científico disponible. En ese sentido, el diplomado buscó generar transformaciones en el ámbito educativo desde los actores que intervienen en las aulas de nuestra casa de estudios.

\section{Fundamentación del diplomado}

El diplomado "Investigar en educación desde la práctica docente" se diseñó desde la teoría sociocultural, al entenderlo como un espacio que busca la negociación del significado de investigación y la apropiación de herramientas teórico- 
metodológicas para su desarrollo, dentro de prácticas educativas reconocidas por los participantes, con la intención de que los contenidos abordados representaran saberes contextualizados y con sentido dentro del ejercicio profesional de los profesores (Guitar, 2011).

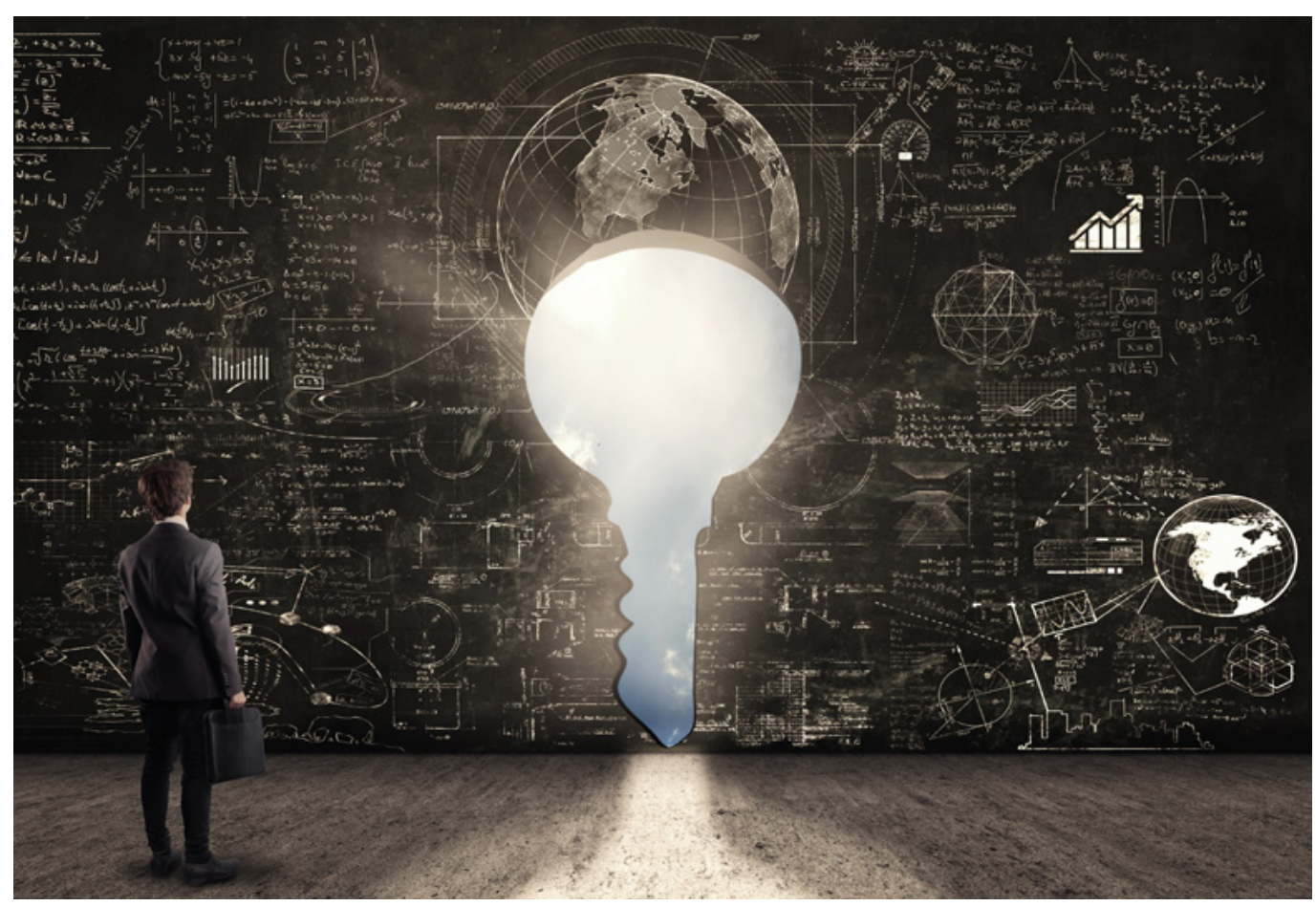

Se asumió el aprendizaje como un proceso multidimensional que involucra pensamiento, afectividad y acción dentro de sus propias prácticas educativas, proporcionando así significado y sentido a los contenidos que se abordaron a lo largo de la formación (Baquero, 2002).

También se consideró que dicho aprendizaje se origina en lo social, dentro un sistema de comunicación entre participantes, a través de la interacción, colaboración y reciprocidad de todos los involucrados en el diplomado. Dicha comunicación resultó relevante, porque posibilitó la construcción conjunta de conocimiento, a partir de las aportaciones de cada uno de los participantes, lo que les permitió pasar de la realización exitosa de actividades vinculadas a la investigación con ayuda de otras personas, al desarrollo de éstas en forma totalmente autónoma, voluntaria e independiente (Chaves, 2001).

Al mismo tiempo, el diplomado buscó que los participantes plantearan el desarrollo de un producto, un protocolo de investigación, desde alguna motivación, inquietud o necesidad que ellos mismos tuvieran para resolver una problemática o mejorar algo de su ejercicio profesional, y que a partir de ello generaran propuestas de investigación que les brindaran posibles soluciones (Díaz Barriga, 2003). 
Por otro lado, las actividades consideradas dentro del diplomado estuvieron organizadas de manera graduada, en función del avance que los profesores iban teniendo respecto al desarrollo de conocimientos, habilidades y actitudes, frente a la complejidad de las situaciones que se abordaron (Leguízamo, Montaño y Villarroel, 2004; Sagástegui, 2004), lo cual les permitió fortalecer también sus valores y compromisos con el proceso de formación y su práctica educativa (Maldonado, 2008; Scruzzi, 2016).

Además, a lo largo de todo el proceso de formación, se buscó que el diplomado se convirtiera en un espacio para la construcción de una comunidad de aprendizaje. Es decir, un foro abierto al diálogo entre participantes, donde todos asumieran un rol activo dentro de las actividades a desarrollar, y pudieran poner en juego sus capacidades a través del trabajo cooperativo; así como que aprendieran a recibir ayuda de sus pares y facilitadores, y a ofrecerla, con la finalidad de enriquecer sus procesos cognitivos e ideas para la creación de proyectos de investigación sólidos, adecuados y útiles dentro de los contextos en que fueron pensados (Gertrudix y García, 2011).

Como estrategia didáctica se utilizó el aprendizaje basado en proyectos $\left(A_{B} P\right)$, con lo que se pretendió fomentar en los participantes entusiasmo por el proceso de investigación, la generación de hipótesis, ideas, reflexiones y discusiones, al tomar en cuenta sus intereses y buscar que aplicaran en todo momento el conocimiento adquirido en el desarrollo de su protocolo de investigación. Lo anterior permitió organizar las actividades en torno a un fin común y fortalecer el compromiso adquirido por ellos dentro del diplomado.

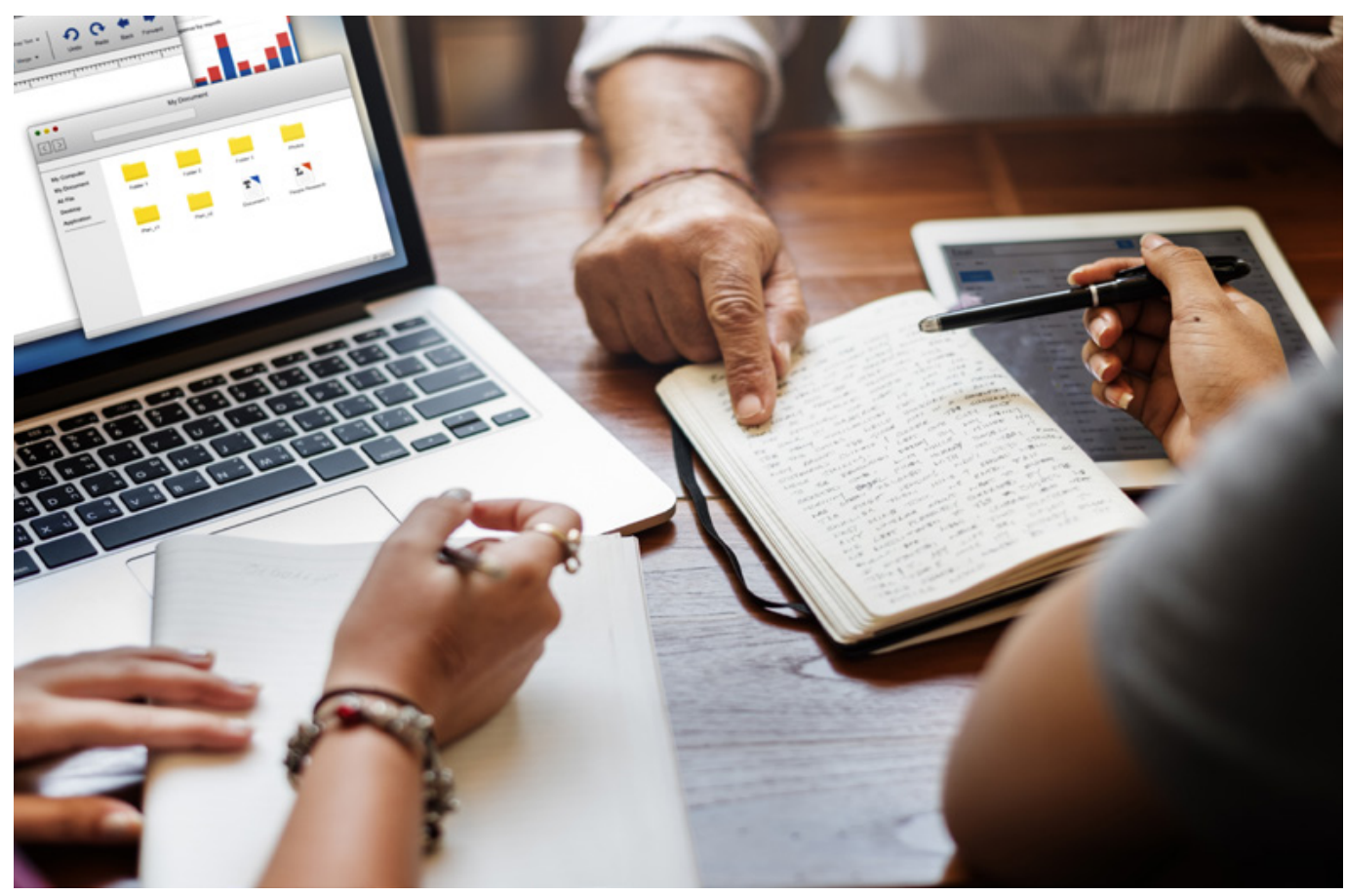


El desarrollo del protocolo de investigación no quedó confinado al término del proceso formativo, sino que constituyó el centro del mismo. Es decir, el proceso de enseñanza no precedió al progreso del protocolo, sino que estuvo integrado en él, de manera que los avances en la construcción de éste fue lo que dio lugar a los aprendizajes más relevantes y significativos.

\section{Estructura del diplomado (St1)}

El contenido estuvo organizado en cinco módulos, a través de los cuales los profesores construyeron gradualmente su protocolo de investigación:

Módulo I. El sentido de mi objeto de investigación.

Módulo Il. Las bases de mi investigación.

Módulo III. Mis fuentes de información.

Módulo IV. Mi forma de investigar.

Módulo $\vee$. Los alcances de mi investigación.

Cada módulo estuvo integrado por temas, y a su vez cada tema contó con secuencias de aprendizaje que se abordaron en sesiones presenciales y de trabajo a distancia.

El diplomado se impartió a lo largo de 15 semanas, del 27 de julio al 7 de diciembre del 2017. Por semana, el tiempo de dedicación programado para los profesores participantes fue de diez horas.

Cada módulo inició y concluyó con una sesión presencial. Por la extensión de los módulos ॥, III y Iv, éstos tuvieron una sesión presencial intermedia. En total fueron 13 sesiones presenciales, siempre programadas los días jueves de 15:00 a 19:00 horas. El resto del trabajo se realizó a distancia.

Durante todo el proceso de formación, los docentes contaron con el apoyo de una asesora, cuya labor principal fue brindarles un acompañamiento personalizado. Agregado a ello, en las sesiones presenciales, se contaron con diferentes facilitadores expertos en cada tema abordado.

Se utilizó el sistema de gestión de aprendizaje Moodle para el trabajo a distancia, y la entrega de avances en el desarrollo se su protocolo. 


\section{La experiencia del diplomado a través de la voz de los participantes}

\section{Los profesores formados}

Para conocer parte de los resultados obtenidos con el desarrollo de diplomado, se aplicó un cuestionario a nueve de los doce docentes que concluyeron el proceso de formación. El propósito fue recoger su experiencia y opinión del diplomado como proceso de formación, en cuanto a diversos ejes: contenidos, materiales, actividades presenciales, actividades a distancia, asesoría y acompañamiento; así como respecto a si el diplomado había cumplido con sus objetivos y expectativas. Los resultados fueron los siguientes:

\section{Contenidos}

Los contenidos fueron considerados adecuados y pertinentes por todos los participantes, la mayoría apreció que también fueron suficientes. En términos generales no quitarían ningún contenido, aunque sí hay sugerencias de agregar nuevos que revelan el interés particular de cada estudiante. Estas sugerencias muestran sus necesidades de formación para continuar con el planteamiento e implementación de su protocolo. Asimismo, señalaron de forma unánime que la organización de los contenidos fue pertinente.

\section{Materiales}

Sólo uno de los docentes participantes consideró que los materiales diseñados de forma original para el diplomado no fueron adecuados ni pertinentes, el resto expresó comentarios de aprobación, entre los que se cuenta que fueron útiles, didácticos, sencillos y precisos.

Respecto a las lecturas y referencias bibliográficas proporcionadas, nuevamente un participante expresó que no fueron adecuadas ni pertinentes; los demás las valoraron positivamente.

\section{Actividades presenciales}

La mayoría de los participantes expresaron comentarios positivos de este tipo de actividades, sólo se recogieron dos comentarios que destacaron áreas de mejora para estos aspectos. El día y hora para las sesiones fue adecuado para todos, salvo para un estudiante que se le dificultaba pedir permiso en su trabajo.

\section{Actividades a distancia}

La mayoría de los profesores formados expresaron comentarios positivos de este tipo de actividades, de la distribución y tiempos de entrega; sólo dos 
comentarios señalan áreas de mejora en estos aspectos. Todos los participantes hicieron sugerencias para mejorar el trabajo a distancia, que en particular se relacionan con la plataforma.

\section{Asesoría y acompañamiento}

Todos los comentarios de los participantes fueron positivos acerca del desempeño de las asesoras en las sesiones presenciales y en el acompañamiento a distancia. También brindaron sugerencias para mejorar esta función y estar más cerca de los estudiantes y sus protocolos.

\section{Cumplimiento de objetivos y expectativas}

Los nueve participantes respondieron que sí se cumplieron los objetivos planteados en el diplomado, sólo en un caso se mencionó que no se cumplieron todas sus expectativas. Para todos será muy útil lo aprendido, y hay consenso en cuanto a que recomendarían el diplomado, excepto en un caso, donde no lo recomendaría totalmente.

Agregado a lo anterior, dentro de la sesión de cierre, los docentes expresaron libremente su percepción como participantes y usuarios de esa propuesta, de tal manera que pudimos contar con información adicional, muy valiosa para evaluar el logro de las metas planteadas para el diplomado.

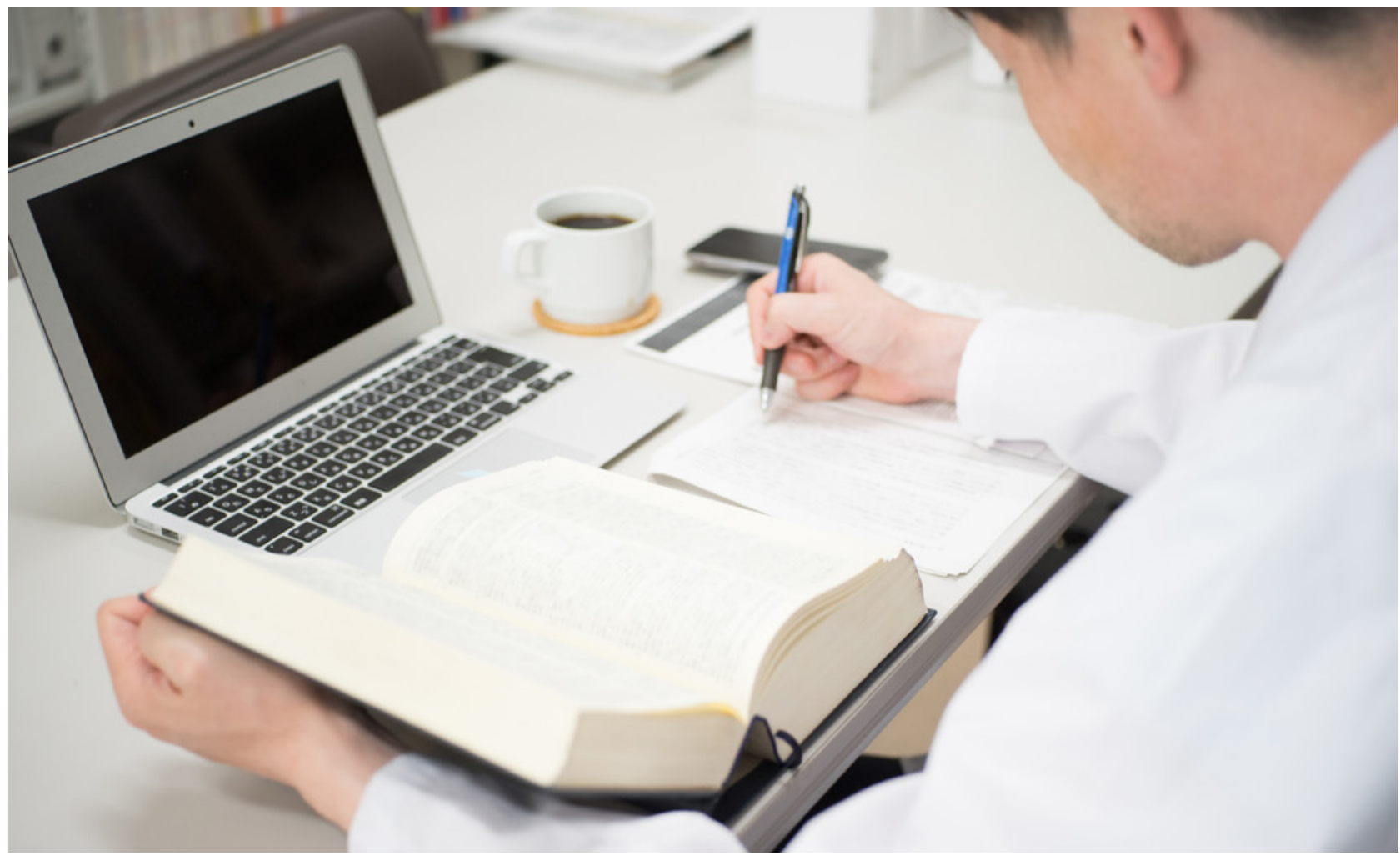


De acuerdo con lo que expresaron quienes cursaron la primera emisión del diplomado, el intercambio que tuvo lugar entre los participantes a lo largo del proceso formativo, tanto en las sesiones presenciales como vía la plataforma, lo consideraron muy enriquecedor y formativo. Al respecto mencionaron asuntos como los siguientes:

[...] esto me pareció muy rico, tan rico como es el ver todas las diferencias que tenemos respecto de nuestros puntos de interés, pero en un ámbito escolar, en un ámbito educativo... en fin, aprender a tolerar las opiniones de otros, las miradas de otros, yo creo que es parte de las habilidades de investigación que tendríamos que aprender (testimonio de docente 1).

[...] sí, yo estoy también bien agradecida, y también obviamente las investigaciones de los compañeros, o sea, es bien interesante, uno no sabe con quién se codea en estos diplomados [risas], pero uno aprende mucho de ellos (testimonio de docente 4).

[...] en mi caso, cuando empecé, lo platicaba con los compañeros, y me decían: "Cálmate, no es una tesis de vida jeh!... Es un simple trabajo de investigación, tienes que acotarlo, centrarlo". Entonces, eso fue lo que logré aquí y además lo enriquecí con todos ustedes compañeras... Me enriquecí tanto con su participación y con la de ustedes [las asesoras] que nos orientaron de manera muy práctica para llegar al resultado que es visible (testimonio de docente 5).

En ese sentido, es posible afirmar que la apuesta en cuanto el trabajo colaborativo del diplomado fue un acierto y los participantes lo consideraron una de las riquezas de esta propuesta formativa.

De igual forma, el construir los contenidos y metodología del diplomado tomando como referente una aproximación práctica hacia su quehacer cotidiano, no sólo tuvo sentido para los participantes, sino que percibieron que se aproximaron a la investigación desde un enfoque que no habían contemplado y eso transformó su visión sobre ésta. Quienes cursaron el diplomado comentaron aspectos como los que se citan a continuación.

[...] para mí eso sí ha sido como que ¡GUAU! ¿no? Yo digo jah! se puede llegar a esto por esta vía, y con resultados muy sólidos, ¿no?, de conocimientos, de herramientas... Lo asumí como un desafío porque era el espacio para pensar en algo que me ha gustado pero nunca tenía tiempo (testimonio de docente 1).

[...] se los agradezco mucho, fue muy cercana esa manera para los docentes que es mi caso que no nos hemos dedicado a la investigación dije "iAy 
de lo que me he estado perdiendo", y bueno, pero nunca es tarde, estamos recuperando experiencias en otros campos, y ahora con estos pasitos, no, iyo estoy encantada! (testimonio de docente 1).

Yo sí también quiero agradecer, para mí fue como descubrir, igual que dice [...] algo que es maravilloso, que de alguna manera, en algunos otros momentos de la vida ha sido como muy azaroso, muy solitario... Y aquí, al menos a mí me ayudaron a poner... como más sistemático el asunto, e ir trazando como un camino, muchas gracias (testimonio de docente 3 ).

Yo considero que este tipo de trabajos, de ustedes, su planeación, la forma como nos llevaron así de la manita fue como... como... siento que va a ser el parteaguas para muchos de nosotros... que aunque ahí estaba [el conocimiento] y entre comillas lo sabíamos, no se había aplicado, entonces, al final de nuestro proyecto se convirtió en algo fundamental y sobre todo... lo que ustedes provocaron, esa experiencia humana que siento que es invaluable (testimonio de docente 7).

[...] en otros cursos que he tomado de investigación se da la cuestión de [que] siempre lo teórico ahí está muy bonito y lo expresan y te ponen a hacer ejercicios sin sentido, no están integrados, no tienen una finalidad, y es una cuestión teórica que no sirve para gran cosa..., la cuestión es cómo ellas lograron que nosotros aplicáramos todo esto en una idea muy difusa que teníamos (testimonio de docente 5).

Finalmente, expresaron la satisfacción o logro que les significa haber concluido con un producto que les parecía lejano de construir. Compartieron que el diplomado les permitió llegar a la elaboración de un protocolo de investigación como se tenía previsto, y que la construcción de éste, mediante la metodología y contenidos dispuestos para su aprendizaje, les brindó más enseñanzas y crecimiento de los que esperaban. En sus palabras:

[...] este diplomado a mí lo que me sirvió, bueno aprendí, por supuesto aprendí, pero también a sentirme más segura, muchísimo más segura, y además no sé... éste es como un nicho así bien bonito donde por fin encuentro las personas que están preparadas para orientarnos para seguir haciendo esa labor que, bueno, a mí por lo menos me enriquece y me pone bien feliz cada vez que estoy en un aula, ¿no?, con los muchachos (testimonio de docente 4).

[...] yo quiero reconocer que si yo me hubiera impuesto hacer esto, en este mismo tiempo sola, no lo hubiera logrado, pero acompañada y con la guía de ustedes [las asesoras] pues más o menos lo fui logrando (testimonio de docente 6). 
[...] me gustaría poder rescatar esos materiales porque yo creo que esta experiencia en nosotros no nos sirve nada más para este protocolo, sino que nos sirve para futuras investigaciones o incluso yo digo que nosotros vamos a poder ya también ayudar a otros que estén en la misma situación que nosotros, y a decirles "no es tan difícil", el camino puede ser éste (testimonio de docente 6).

\section{Las asesoras del diplomado}

Las tres asesoras que impartieron y brindaron el acompañamiento durante el diplomado compartieron las oportunidades, desafíos, aciertos y aprendizajes que derivaron de su experiencia.

En cuanto a las oportunidades, consideraron que la principal fue la puesta en marcha de una alternativa para formar investigadores en educación, que implicó tomar como punto de partida los incidentes críticos de la práctica docente; así como, la oportunidad de compartir la experiencia con la que cuenta el equipo de CODEIC en el ejercicio simultáneo de la investigación y la docencia.

[...] representó el primer desarrollo realizado por el equipo de la subdirección, el cual está conformado por profesionales de diferentes disciplinas. Fue muy rico, debido a la formación y experiencia que tienen mis compañeras de trabajo en el ámbito educativo, creo que realmente logramos construir un diplomado donde se ponía lo mejor de cada una en todos los sentidos, conocer el nivel de compromiso y responsabilidad que asumen en el trabajo, y saber que lo compartimos, también fue muy grato (testimonio de asesora 1).

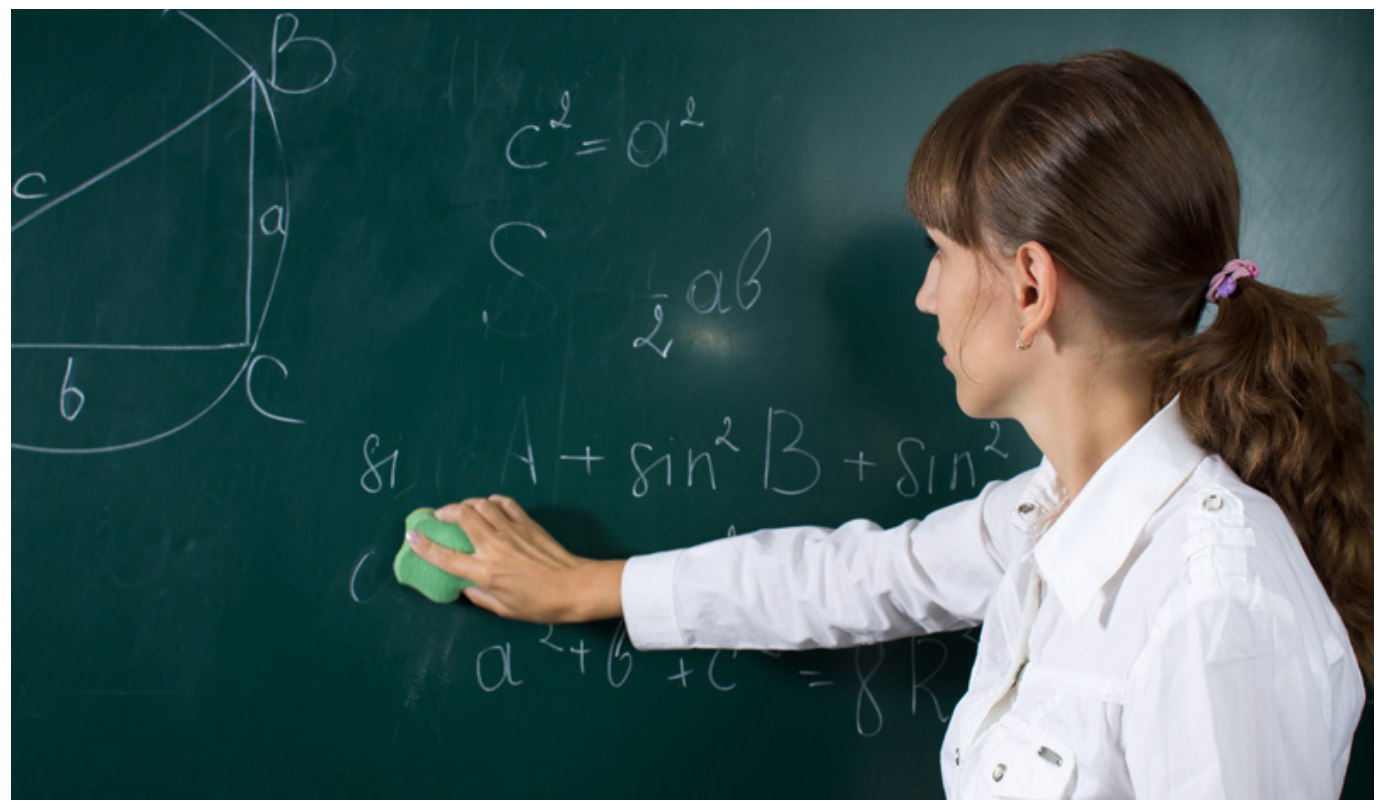


Participar en el diplomado también les significó desafíos, el principal fue desarrollar un proceso formativo basado en nociones de investigación y docencia como procesos complementarios, vinculado a mejoras educativas.

[...] el planteamiento, dado que implicó defender la propuesta desde el inicio, debido a que representaba un proyecto de formación distinta, que iniciaba por reconocer la importancia de la labor del docente, así como la experiencia que los profesores tienen, útil para el desarrollo de investigaciones en educación que realmente atiendan sus necesidades, es decir, se pensó en el docente como un constructor de conocimiento, no sólo como un consumidor (testimonio de asesora 1).

Fue un gran reto por ser la primera vez que se diseñaba un diplomado así, pensado para que los profesores Universitarios realizaran investigación en educación desde su experiencia en la docencia (testimonio de asesora 2).

El desafío de poner en práctica cómo la investigación puede apoyar la mejora educativa si se le concibe desde el propio quehacer docente, lo que implicó, en algunas ocasiones, ir más allá de cómo se conciben tradicionalmente las tareas de investigación en la universidad. Considero que no se necesita dejar de ser docente para ser investigador, pues ambos quehaceres pueden complementarse. Ahora bien, al igual que en la docencia, para desarrollar investigación es necesario formarse, desarrollar algunas habilidades, porque tampoco es una tarea de "generación espontánea" (testimonio de asesora 3).

Otro desafío que vivieron fue el institucional, pues implicó vincular dos dependencias, CODEIC, de reciente creación, y la Coordinación de Universidad Abierta y Educación a Distancia (CUAED), ya consolidada y con procedimientos bien establecidos. Hay que añadir que este proyecto tenía sobre sí múltiples expectativas, tanto por ser el diplomado pionero de la Subdirección de Investigación en Educación, como también el primero de codeıc en colaboración con otra dependencia de la universidad.

[...] por el vínculo que se estableció entre dependencias (CUAED) y la forma de trabajo que se implementó, todo ello significó grandes aprendizajes y permitió que el diseño del diplomado fuera un acierto (testimonio de asesora 2).

Finalmente, dentro de los retos, las asesoras no pudieron dejar de mencionar lo que implicó el sismo del 19 de septiembre de 2017, no sólo para esta actividad académica, sino para gran cantidad de procesos formativos, ya que la continuidad de algunos participantes se vio comprometida, tanto por asuntos materiales, como familiares y psicológicos. 
Otro de los retos que enfrentamos fue la condición de trabajo, y de vida (para todos), que representó el sismo del 19 de septiembre, fue necesario hacer ajustes y modificar un poco el plan de trabajo que se tenía previsto, pero nuevamente el reto fue sorteado con resultados satisfactorios para todos (testimonio de asesora 2).

Por otro lado, también les interesó hablar de los que a su juicio son los aciertos de la experiencia, sobre todo con la intención de aportar elementos que puedan inspirar a quienes estén interesados en el diseño de oferta formativa como la que aquí se muestra. En primer lugar, confirmaron que la construcción de un protocolo de investigación en educación no es un quehacer en solitario, es un proceso que se transita mejor cuando se cuenta con el acompañamiento de un asesor y se somete a la realimentación entre pares.

Considero que la investigación no es un quehacer en solitario, y al planear este diplomado pudimos comprobar que la investigación es mejor si se desarrolla acompañado por un tutor y si se cuenta con la mirada de pares que también están interesados en ella, y que pueden compartir con uno sus experiencias. El acompañamiento es particularmente importante para el trabajo semipresencial (testimonio de asesora 3).

También encontraron que fue un acierto construir un espacio horizontal entre docentes de diversos niveles educativos, áreas de formación y años de experiencia; eso contribuyó a que el diplomado fuera útil y adquiriera sentido para el profesorado. Reconocieron que la formación, experiencia y trayectoria de todos los participantes son fuentes de aprendizaje invaluables. La experiencia convocó a diversos miembros de la comunidad universitaria a trabajar en pro de la mejora de la institución de la que forman parte, ayudó a estrechar los vínculos y a dar inicio a una red de docentes que investigan desde su práctica.

Y ha sido una gran satisfacción experimentar el acierto que fue aquella propuesta, tanto conceptual como metodológica, para que los docentes universitarios, de los diferentes niveles educativos, en un espacio horizontal, realizaran investigación en educación desde su práctica educativa, generaran protocolos de investigación sobre temáticas que atañen a los profesores en las aulas y que se traducen en mejoras para la universidad [...] Además, el vínculo que se estableció entre los participantes permitió que se conformara una red en donde el intercambio de conocimiento y experiencias continúa vigente aun concluido el diplomado; finalmente la satisfacción más grande es haber creado, en conjunto con el equipo de trabajo, una propuesta educativa útil y con mucho sentido para el profesorado en donde el fin último es la mejora de la educación universitaria, de la experiencia docente y de los alumnos que cursan su formación en las aulas de la UNAM (testimonio de asesora 2). 
Ha sido también importante el logro de acuerdos interinstitucionales para alcanzar objetivos conjuntos, gracias a la flexibilidad y aprendizaje obtenidos por la relación CODEIC-CUAED.

Desde la forma de trabajo entre dependencias de la UNAM, la colaboración que existe en los grupos de trabajo, que aunque procedan de dependencias diferentes apuestan a lo mismo: una oferta educativa en pro de la mejora universitaria (testimonio de asesora 2).

Además, en el equipo de trabajo, fue muy valioso el construir cada fase del proyecto con compromiso y corresponsabilidad, así como involucrarse todas en el desarrollo de múltiples tareas en cada etapa.

[...] el desarrollo de diferentes actividades al mismo tiempo: diseño instruccional, elaboración de materiales, impartición del diplomado y seguimiento a cada uno de los participantes con sus proyectos (testimonio de asesora 1).

La creación, construcción, impartición y asesorías a los participantes se daba de manera simultánea y el tiempo de cada una de las actividades ya estaba establecido; la atención a esas actividades además de las responsabilidades adquiridas con otros proyectos de la subdirección obligó a que el trabajo fuera muy organizado, y al estar en constante comunicación con el equipo de trabajo permitió que el reto fuera enfrentado de manera muy positiva y con resultados gratificantes para todos (o al menos la mayoría) (testimonio de asesora 2).

El reto de diseñar a la par que impartir fue importante. Me retó el tener que diseñar materiales exprofeso que incluyeran diferentes perspectivas de investigación para atender la diversidad de temáticas de interés de los asistentes y sus múltiples áreas y niveles; así como que tuvieran como principal característica ser lo suficientemente concretos y con un lenguaje comprensible para todos (testimonio de asesora 3).

Finalmente, hablaron de los aprendizajes que deja esta experiencia. En un ámbito personal, fortalecieron relaciones al interior del equipo y con los participantes del diplomado; aprendieron que es necesario defender los proyectos, no claudicar en las dificultades iniciales, y sí construir alternativas, ser flexibles y reorganizarse; también se dieron cuenta de sus capacidades y de cómo la organización hace factible que un equipo pequeño pueda trabajar eficientemente en diversas etapas de un mismo proyecto. En colectivo, fueron testigos de cómo el trabajo interdisciplinario rinde frutos, y la importancia de apostar por el trabajo colaborativo. Institucionalmente, aprendieron a situarse al servicio de los docentes para poder acompañarlos en el desarrollo de sus protocolos, a flexibilizar la plataforma Moodle con la finalidad de adaptarla a las necesidades 
de sus usuarios, a desarrollar materiales que comunicaran elementos de investigación claves en un lenguaje sencillo y útil para el ejercicio de la investigación, en aquellos que apenas comienzan a desarrollarla.

Tomar conciencia de que siempre requeriré fortalecer mis conocimientos y habilidades, en este caso, en el desarrollo de investigación en el campo educativo, darme cuenta que es muy poco lo que sé, y mucho lo que aún me queda por aprender (testimonio de asesora 1).

La riqueza de los proyectos de los participantes, las problemáticas o situaciones a las que se enfrentan y la forma en la que las atienden también representaron aprendizajes sobre los fenómenos que ocurren en nuestra universidad, y caracteriza en cierta medida a los docentes y estudiantes que están en sus aulas (testimonio de asesora 2).

Hay que escuchar a los participantes y hacer flexible la plataforma para cubrir las necesidades del grupo (testimonio de asesora 3).

Valoré la voz de los docentes, aprendí a escuchar sus necesidades y construir desde ahí, ellos son los protagonistas, tienen un papel fundamental en el ámbito educativo, y lo menos que podemos hacer es estar a su servicio para apoyarlos y acompañarlos (testimonio de asesora 1).

Tuve la oportunidad de continuar construyendo y aprendiendo de mis compañeras de trabajo, cada experiencia laboral y proyectos en los que colaboramos son una fuente de aprendizaje importante para mí (testimonio de asesora 2).

He aprendido que el trabajo en equipo interdisciplinario rinde grandes frutos (testimonio de asesora 3).

Después de esta experiencia, confirmo que como personas y profesionales debemos aferrarnos a nuestras ideas, no claudicar a la primera (testimonio de asesora 1).

\section{Conclusiones}

De acuerdo con los datos anteriores, se puede concluir que los profesores que participaron y se formaron dentro del diplomado valoraron positivamente los diversos elementos considerados: contenidos, materiales, actividades presenciales, asesoría y acompañamiento, y el cumplimiento de objetivos y expectativas. Hay un 
Fotografía por Francisco Parra, en: Gaceta UNAM, No. 4,940, 15 febrero 2018. aspecto a mejorar, y éste se encuentra vinculado con el trabajo y las actividades a distancia; por un lado, mejorar la estructura y organización de los elementos que contiene la plataforma Moodle, y por otro, reconsiderar el número de actividades que están planteadas para cada módulo.

Lo anterior, agregado a los comentarios expresados de manera libre por los mismos participantes, hace pensar que los propósitos con los que surgió el diplomado fueron cubiertos, y que la perspectiva desde donde fue planteado el programa para abordar el desarrollo de investigación en educación vinculado con el ejercicio de la docencia representó un gran acierto, dado que no sólo tuvo sentido para quienes lo cursaron, sino que transformó positivamente su visión sobre la investigación. El ejercicio de la investigación se convirtió en una herramienta útil y valiosa para su quehacer como docentes, y además les brindó satisfacciones y crecimiento tanto profesional como personalmente.

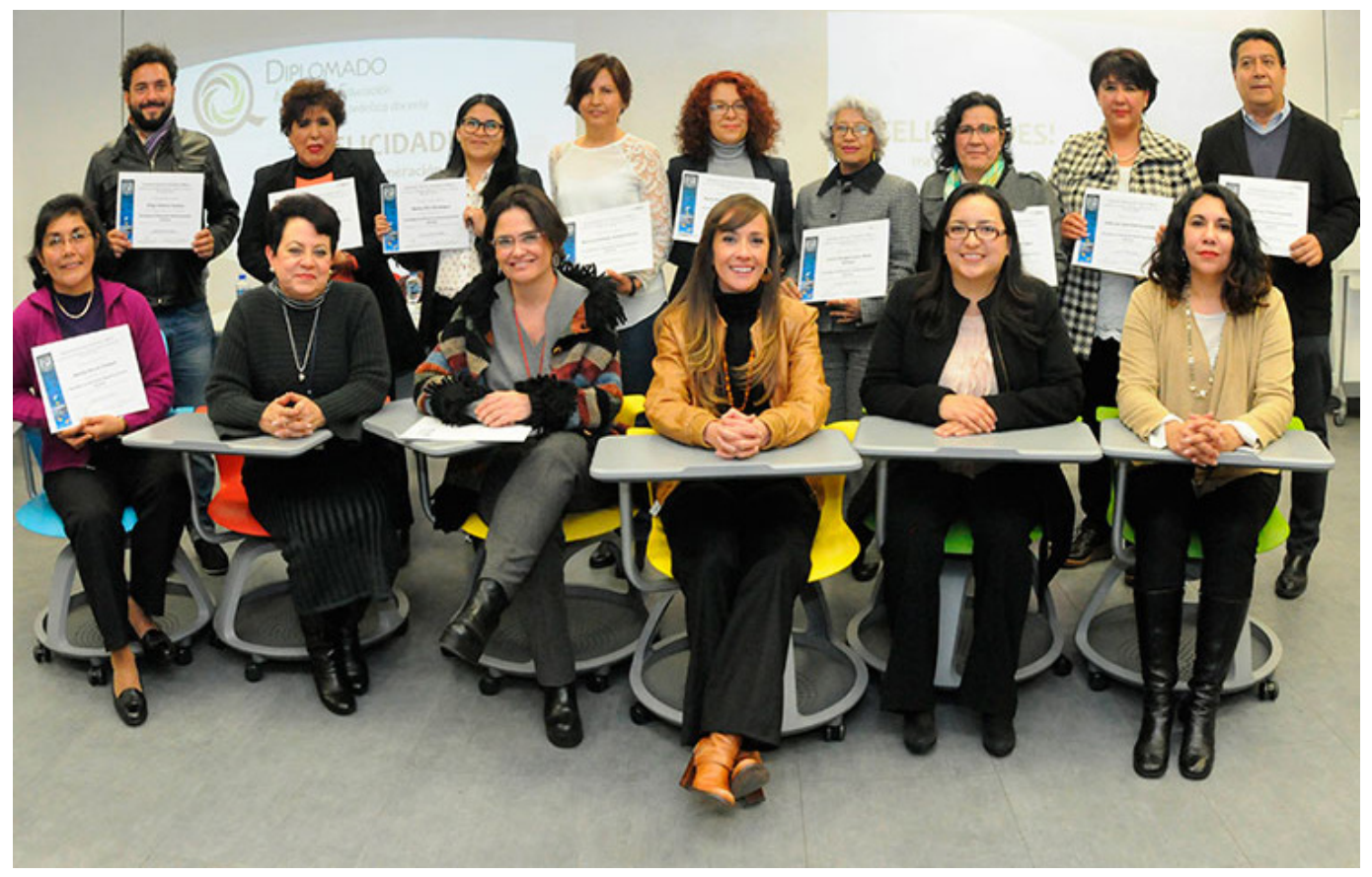

Para las asesoras, el diplomado también aportó aprendizajes muy significativos y gratos, puesto que les permitió acercarse a cada entidad de la UNAM, a través de los profesores formados, y darse cuenta que en cada dependencia de la institución se encuentran personas que apuestan por la mejora educativa, y que es de gran valía haber podido compartir experiencias con ellos.

Además, les muestra con claridad la importancia de fortalecer sus conocimientos y habilidades en materia de investigación en educación, para ofrecer a los investigadores en formación nuevas perspectivas y enfoques, en aras de ayudarlos a introducirse en ese campo dinámico y complejo. Asimismo, la experiencia las invita a mantener una actitud abierta a la formación continua, 
empatía, comprensión y apoyo a los pares investigadores y docentes dentro y fuera de la UNAM.

\section{Referencias}

- Baquero, R. (2002). Del experimento escolar a la experiencia educativa. La transmisión educativa desde una perspectiva psicológica situacional. Perfiles Educativos, 24(98), 57-75.

* Chaves, A. (2001). Implicaciones educativas de la teoría sociocultural de Vigotsky. Revista Educación, 25(2), 59-65. Dol: https://doi.org/10.15517/revedu.v25i2.3581

* Díaz Barriga, F. (2003). Cognición situada y estrategias para el aprendizaje significativo. Revista Electrónica de Investigación Educativa, 5(2), 105-117.

* Gertrudix, F., y García, F. (2011). Aprendizaje situado y cooperativo en educacíon superior: una experiencia con equipos de trabajo en ciencias de la comunicacíon. Revista Linhas, 12(2), 18-30.

* Guitar, E. (2011). Aplicaciones contemporáneas de la teoría vygotskiana en educación. Revista de Educación y Desarrollo Social, 5(1), 95-113.

- Leguízamo, A., Montaño, N. y Villarroel, G. (2004). Una experiencia de aprendizaje situado: laboratorio de desarrollo de software. Recuperado de: http://www.Imi.ub.es/ edutec2004/pdf/156.pdf

* Maldonado, M. (2008). Aprendizaje basado en proyectos colaborativos. Una experiencia en educacion superior. Laurus, 14, 158-180.

* Sagástegui, D. (2004). Una apuesta por la cultura: el aprendizaje situado. Revista Electrónica Sinéctica, 24, 30-39. Recuperado de: http://www.redalyc.org/resumen. oa?id=99815918005

* Scruzzi, G. (2016). El aprendizaje situado como instrumento de promoción del compromiso social. Didac, 67, 33-54.

\section{Cómo citar este artículo}

- Lara Gutiérrez, Yazmín Alejandra, Olvera López, Adriana y Pompa Mansilla, Maura (2018). Los docentes como investigadores de su práctica: una experiencia de formación. Revista Digital Universitaria (RDU). Vol. 19, núm. 4 julio-agosto. DOI: http:// doi.org/10.22201/codeic.16076079e.2018.v19n4.a5. 